\title{
Direct monitoring pressure overload predicts cardiac hypertrophy in mice
}

\author{
Sheng Zhong Duan ${ }^{1}$, Christine Y Ivashchenko ${ }^{1}$, Steven E Whitesall ${ }^{1}$, \\ Louis G D'Alecy ${ }^{1,2,3}$ and Richard M Mortensen ${ }^{1,4,5}$ \\ ${ }^{1}$ Department of Molecular and Integrative Physiology, University of Michigan Medical School, \\ 7744 Med. Sci. II, 1150 W. Med. Ctr. Dr., Ann Arbor, MI 48109-0622, USA \\ ${ }^{2}$ Department of Surgery (Vascular), University of Michigan Medical School, Ann Arbor, MI, \\ USA \\ ${ }^{3}$ Department of Surgery, William Beaumont Hospital, Royal Oak, MI, USA \\ ${ }^{4}$ Department of Pharmacology, University of Michigan Medical School, Ann Arbor, MI, USA \\ ${ }^{5}$ Department of Internal Medicine, Metabolism Endocrinology and Diabetes Division, University \\ of Michigan Medical School, Ann Arbor, MI, USA \\ E-mail: rmort@umich.edu
}

Received 18 May 2007, accepted for publication 12 September 2007

Published 5 October 2007

Online at stacks.iop.org/PM/28/1329

\begin{abstract}
Pressure overload (POL) is a classical model for studying cardiac hypertrophy, but there has been no direct measure of hemodynamics in a conscious ambulatory mouse model of POL. We used abdominal aortic constriction to produce POL and radiotelemetry to measure the blood pressure and heart rate for three weeks. The cardiac size correlated with the systolic pressure in the last week is better than other hemodynamic parameters. Cardiac fibrosis was more correlated to the cardiac size than to the systolic pressure. The expression of the cardiac genes that are typically associated with cardiac hypertrophy was correlated with both cardiac size and systolic pressure. In conclusion, the systolic pressure is the major determinant of cardiac hypertrophy in the murine POL model. In contrast, cardiac fibrosis shows the influence of other factors besides systolic pressure. The combination of the POL model with continuous direct measurements of hemodynamics represents a significant technological advance and will lead to an extended usefulness of POL methodologically.
\end{abstract}

Keywords: cardiac hypertrophy, cardiac gene expression, fibrosis, pressure overload 


\section{Introduction}

The pressure overload (POL) model was introduced to study cardiac hypertrophy as early as the 1950s (Young et al 1950, Alexander 1953). This model, including both transverse aortic constriction and abdominal aortic constriction, was adapted to mice and has been widely used in cardiovascular research (Rockman et al 1991, De Windt et al 2001, Asakawa et al 2002, McMullen et al 2003, Liao et al 2004, De Acetis et al 2005, Takimoto et al 2005). To produce the POL the aorta is commonly constricted by tying a suture against a needle to standardize the degree of restriction. Although Rockman et al measured the carotid pressure acutely after POL (Rockman et al 1991) and the pressure drop across the constriction at the end of POL (Esposito et al 2002) in anesthetized mice, POL has not been monitored in conscious ambulatory mice. Even with the same size of needles, variation in constriction and cardiac hypertrophy can be profound (Rockman et al 1991). These differences may be due to variations in surgical technique leading to different degrees of POL with the same method. Monitoring the degree of POL after aortic constriction may allow for the appropriate weighing of these variations. Cardiac fibrosis is highly associated with POL (Brede et al 2002, McMullen et al 2003, Liao et al 2004, De Acetis et al 2005), but the correlation between fibrosis and hypertrophy or POL has not been fully investigated. Responding to POL, hearts adapt by altering the gene expression, including embryonic, contractile or cytoskeletal, calcium-handling, collective tissue and cardiac metabolism genes (Schoenfeld et al 1998, Hoshijima and Chien 2002, Wagner et al 2004). It is not fully understood to what extent POL or hypertrophy may correlate with these changes in the cardiac gene expression.

In this study, we used the state-of-the-art radiotelemetry to directly and continuously monitor the POL-induced hemodynamic changes (including systolic, diastolic, mean arterial pressure and heart rate) $24 \mathrm{~h}$ a day (Whitesall et al 2004) over 21 days during the progression of cardiac hypertrophy after aortic constriction. We were therefore able to test the correlation of cardiac hypertrophy and various hemodynamic consequences of aortic constriction in order to establish the parameter that is most important in determining the degree of hypertrophy. Further, we evaluated the extent of correlation between cardiac hypertrophy/POL and fibrosis, and between cardiac hypertrophy/POL and cardiac gene expression.

\section{Materials and methods}

Reagents. All reagents were purchased from Sigma-Aldrich (St Louis, Missouri, USA) unless specifically indicated.

Animals. Two months old (20-25 g) male C57Bl/6J mice were purchased from the Jackson Laboratory (Bar Harbor, ME, USA), housed in a 12:12-hour light-dark cycle, fed with standard rodent chow, and given drinking water ad libitum. The animals were cared for in accordance with the Guide for the Care and Use of Laboratory Animals (1996, published by National Academy Press, 2101 Constitution Ave NW, Washington, DC 20055, USA). All animal protocols were approved by the University Committee on Use and Care of Animals of the University of Michigan.

Pressure overload. Abdominal aortic constriction (De Windt et al 2001, Wettschureck et al 2001) was performed to induce pressure overload in mice. Briefly, the mice were anesthetized with $2 \%$ isoflurane inhalation and midline abdominal incisions were made to expose the abdominal aortas. Silk sutures (7-0) were used to ligate aortas against blunted needles with different sizes, $25 \mathrm{G}, 26 \mathrm{G}$ or $27 \mathrm{G}$. The needles were then removed immediately to restore lumens with different stenotic aortic orifices. The abdominal cavity, muscles and skin were 
closed layer by layer with 5-0 silk sutures. For sham-operated mice, silk sutures were passed under aortas and then removed; therefore no constrictions to aortas were performed ( $n=3$ for sham and $27 \mathrm{G}$ groups, $n=4$ for $25 \mathrm{G}$ and $26 \mathrm{G}$ groups).

Direct measurement of $P O L$ by radiotelemetry. One week after abdominal aortic constriction or sham operation, radiotelemetry implants (PA-C20, Data Sciences International; St. Paul, MN, USA) were placed and the pulsatile arterial pressure in the arch of the aorta and hence POL was measured (Whitesall et al 2004). Briefly, the mice were anesthetized with $2 \%$ isoflurane inhalation. The left common carotid was cannulated and the body of the implant was secured in abdominal cavity. Data were collected immediately after implantations for three weeks until the endpoint of experiments at which mice were killed for cardiac phenotypic analyses. Average systolic pressure, diastolic pressure, mean arterial pressure (MAP) and heart rate $(\mathrm{HR})$ were obtained by averaging the data of all measured periods (2-4 weeks) or week 4 only. The pulse pressure was calculated by subtracting the diastolic pressure from the systolic pressure. The rate-pressure product was determined by the product of systolic pressure and HR.

Cardiac hypertrophy estimation. Four weeks after aortic constriction or sham operation, the cardiac size was measured as before (Duan et al 2005). Briefly, the mice were weighed and anesthetized. The hearts were removed and ventricles were dissected for weighing. The ventricular weight to body weight ratio $\left(\mathrm{VW} / \mathrm{BW}, \mathrm{mg} \mathrm{g}^{-1}\right.$ ) was used as an indicator of the cardiac size. The parts of ventricles were then snap frozen in liquid nitrogen for further analyses.

Histologic analysis. The parts of ventricles were fixed in formalin and microtome sections were stained with hematoxylin and eosin (H\&E) and Masson-trichrome (Asakawa et al 2002, Nishida et al 2004, Duan et al 2005). Fibrosis was determined by measuring collagen staining using NIH Image J 1.31V (National Institutes of Health, Bethesda, MD, USA) and expressed as a percentage of staining (green) areas to the total areas examined.

Expression of cardiac genes. The following genes were assayed using reverse transcription quantitative PCR (RT-QPCR) (Schoenfeld et al 1998, Duan et al 2005): type III collagen (Coll III), atrial natriuretic peptide (ANP), $\alpha$-myosin heavy chain $(\alpha$-MHC), $\beta$-myosin heavy chain ( $\beta$-MHC), $\alpha$-skeletal actin (Sk-Actin) and phospholamban (PLBN). SYBR green was used as a universal fluorescent probe and glyceraldehyde-3-phosphate dehydrogenase (GAPDH) was used as an endogenous control.

Statistical analysis. The results were analyzed using stepwise multiple regression (SPSS 15.0 from SPSS Inc., Chicago, IL, USA) or linear regression (Prism from GraphPad Software, Inc., San Diego, CA, USA). The correlations were considered significant if the probability values were $\leqslant 0.05$.

\section{Results}

Cardiac hypertrophy correlates with POL. Preliminary experiments showed more than $75 \%$ mortality if radiotelemetry implantations were performed first or simultaneously with the aortic constrictions. We therefore performed the aortic constrictions a week before the implantations, which gave a less than $15 \%$ mortality. Representative $24 \mathrm{~h}$ pressures (systolic, diastolic and MAP) showed that the smaller the diameter of the needle used (tighter constriction) the greater the pressure (figure 1, table 1) and cardiac size (VW/BW) (table 1) were. Sham group had comparable VW/BW with non-operated regular male C57Bl/6J mice $(3.79 \pm 0.06$ versus $3.81 \pm 0.11, P>0.05)$. Groups of $25 \mathrm{G}, 26 \mathrm{G}$ 

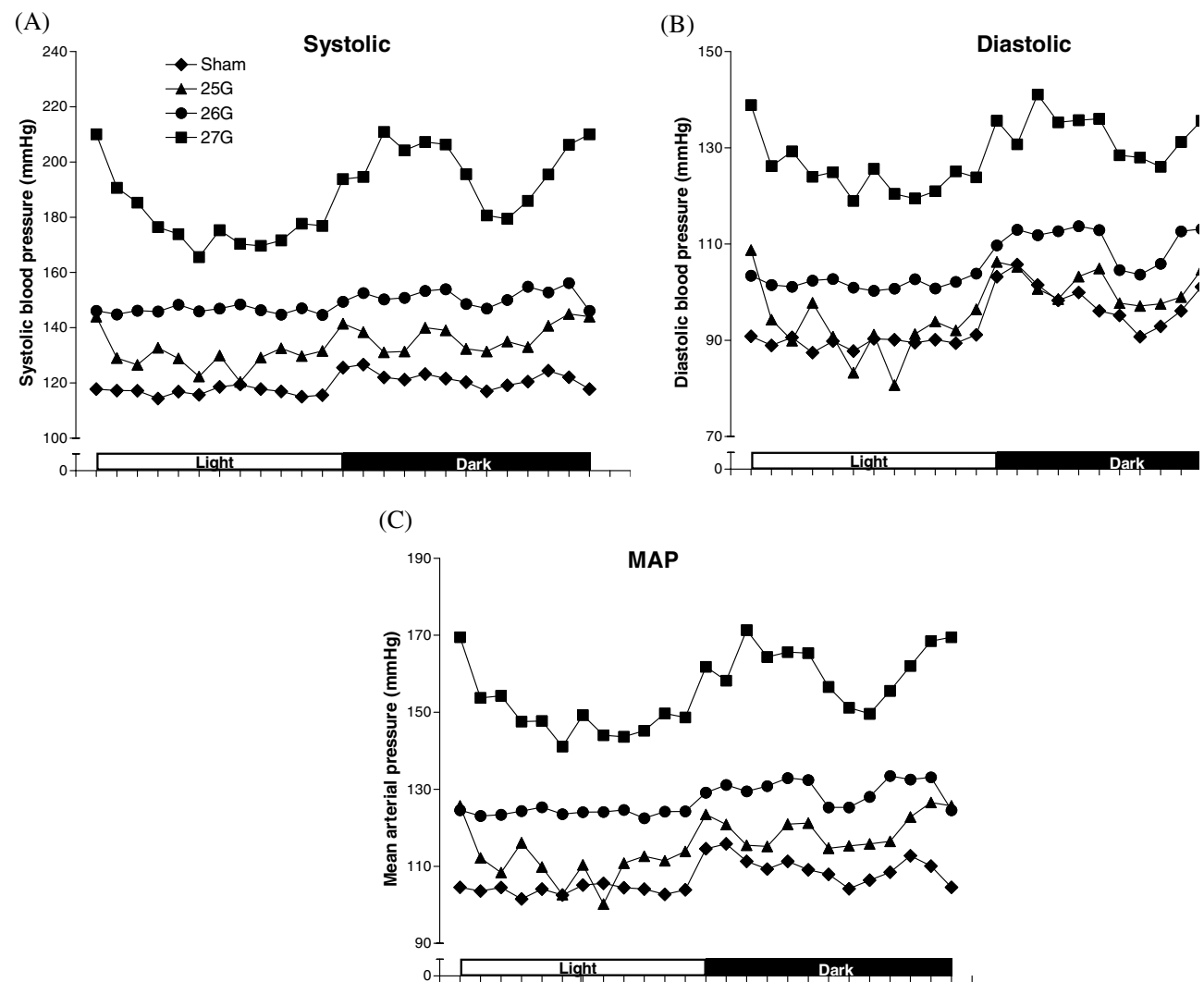

Figure 1. POL monitoring: (A) representative systolic pressure, (B) representative diastolic pressure and $(\mathrm{C})$ representative MAP of sham-operated mice and mice that underwent abdominal aortic constriction with $25 \mathrm{G}, 26 \mathrm{G}$ or $27 \mathrm{G}$ needles. Average pressures of the week 4 were used and graphed using 12:12-hour light-dark cycle.

and $27 \mathrm{G}$ all had a significantly greater $\mathrm{VW} / \mathrm{BW}$ than the sham group $(P<0.01$ for all comparisons). Stepwise multiple regression analyses showed that the week-4 average systolic pressure correlated with $\mathrm{VW} / \mathrm{BW}$ better than the other hemodynamic parameters (diastolic pressure, pulse pressure, MAP, HR and rate-pressure product) did (table 2). More complete analyses revealed that VW/BW correlated better with pressures during week-4 after aortic constriction than with those of all measured periods (total) (table 2). Week-4 systolic pressure was determined as the predictor of VW/BW by stepwise multiple regression.

The significant correlation between week-4 systolic pressure and VW/BW was further demonstrated by a linear regression analysis (figure 2(A)). One of the three mice in the $27 \mathrm{G}$ group had a much smaller increase in $\mathrm{VW} / \mathrm{BW}$ compared with the rest of the group (figure 2(A), indicated by an arrow). However, its systolic pressure was also lower and was proportional to its $\mathrm{VW} / \mathrm{BW}$ (figure 2(A)), demonstrating a tight correlation. Because there was an obvious gap between the $27 \mathrm{G}$ group and the other groups, we performed the linear regression analysis without the $27 \mathrm{G}$ group and found that $\mathrm{VW} / \mathrm{BW}$ still significantly correlated with the week-4 average systolic pressure (figure 2(B)). The week-4 average systolic pressure was used for subsequent correlation studies. 
Table 1. Summary of cardiac hypertrophy and average hemodynamic parameters.

\begin{tabular}{|c|c|c|c|c|c|c|c|c|c|c|c|c|c|}
\hline Group & $\begin{array}{l}\text { VW/ } \\
\text { BW }\end{array}$ & Wk-4 SP & Wk-4 DP & Wk-4 pulse & Wk-4 MAP & Wk-4 HR & Wk-4 RPP & $\begin{array}{l}\text { Total } \\
\text { SP }\end{array}$ & $\begin{array}{l}\text { Total } \\
\text { DP }\end{array}$ & $\begin{array}{l}\text { Total } \\
\text { pulse }\end{array}$ & $\begin{array}{l}\text { Total } \\
\text { MAP }\end{array}$ & $\begin{array}{l}\text { Total } \\
\text { HR }\end{array}$ & $\begin{array}{l}\text { Total } \\
\text { RPP }\end{array}$ \\
\hline Sham & 3.86 & 125.03 & 107.62 & 17.41 & 115.74 & 539.25 & 67421.48 & 128.38 & 110.74 & 17.64 & 118.83 & 529.62 & 67994.45 \\
\hline Sham & 3.73 & 121.99 & 119.01 & 2.98 & 123.53 & 475.90 & 58054.51 & 159.47 & 140.39 & 19.08 & 146.31 & 504.98 & 80527.68 \\
\hline Sham & 3.77 & 119.42 & 93.96 & 25.46 & 107.00 & 546.63 & 65278.95 & 128.20 & 104.03 & 24.17 & 116.14 & 580.58 & 74431.65 \\
\hline $25 \mathrm{G}$ & 4.09 & 138.71 & 112.30 & 26.41 & 124.59 & 580.62 & 80536.48 & 134.91 & 106.94 & 27.96 & 120.55 & 516.53 & 69682.21 \\
\hline $25 \mathrm{G}$ & 4.09 & 133.16 & 97.29 & 35.87 & 115.13 & 571.19 & 76058.77 & 134.85 & 99.67 & 35.17 & 117.11 & 531.70 & 71698.52 \\
\hline $25 \mathrm{G}$ & 4.17 & 143.60 & 132.34 & 11.27 & 137.49 & 454.89 & 65323.61 & 158.57 & 147.66 & 10.90 & 152.41 & 448.86 & 71173.94 \\
\hline $25 \mathrm{G}$ & 4.48 & 141.18 & 110.51 & 30.67 & 125.89 & 566.41 & 79967.80 & 142.73 & 112.03 & 30.70 & 127.56 & 558.57 & 79725.69 \\
\hline $26 \mathrm{G}$ & 4.94 & 148.99 & 106.25 & 42.74 & 127.18 & 576.52 & 85892.97 & 140.12 & 103.85 & 36.27 & 121.88 & 571.05 & 80015.87 \\
\hline $26 \mathrm{G}$ & 4.71 & 146.33 & 107.09 & 39.24 & 119.59 & 503.33 & 73652.44 & 142.09 & 105.94 & 36.15 & 123.35 & 516.84 & 73438.98 \\
\hline $26 \mathrm{G}$ & 4.55 & 135.37 & 102.51 & 32.86 & 118.93 & 506.98 & 68630.93 & 137.84 & 105.58 & 32.26 & 121.78 & 539.16 & 74319.23 \\
\hline $26 \mathrm{G}$ & 4.40 & 133.55 & 102.64 & 30.91 & 118.11 & 508.95 & 67971.95 & 136.22 & 107.53 & 28.69 & 121.72 & 539.16 & 73443.30 \\
\hline $27 \mathrm{G}$ & 6.13 & 187.67 & 129.23 & 58.44 & 155.21 & 576.52 & 108196.60 & 169.15 & 127.46 & 41.69 & 145.89 & 538.31 & 91053.94 \\
\hline $27 \mathrm{G}$ & 6.33 & 207.79 & 196.90 & 10.89 & 203.25 & 455.66 & 94680.70 & 205.62 & 196.48 & 9.14 & 200.41 & 481.58 & 99021.79 \\
\hline $27 \mathrm{G}$ & 5.28 & 149.42 & 128.62 & 20.80 & 136.51 & 487.03 & 72771.79 & 144.79 & 124.63 & 20.16 & 132.79 & 476.55 & 68997.33 \\
\hline
\end{tabular}

$\mathrm{VW} / \mathrm{BW}$, ventricular weight/body weight $\left(\mathrm{mg} \mathrm{g}^{-1}\right)$;

SP, systolic pressure $(\mathrm{mmHg})$;

$\mathrm{DP}$, diastolic pressure $(\mathrm{mmHg})$

Pulse, pulse pressure $(\mathrm{mmHg})$;

MAP, mean arterial pressure $(\mathrm{mmHg})$

RR, heart rate (beats $\mathrm{min}^{-1}$ ),

RPP, rate-pressure product $\left(\mathrm{mmHg} \times\right.$ beats $\left.\mathrm{min}^{-1}\right)$

Wk: week. 

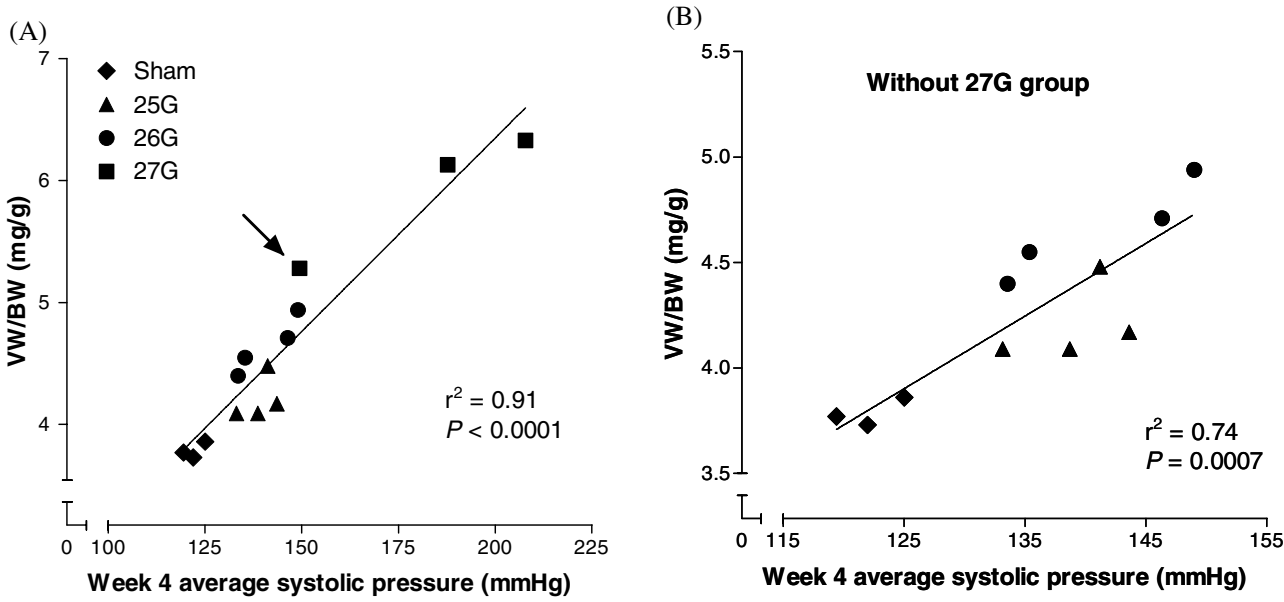

Figure 2. Correlation between cardiac hypertrophy and week-4 systolic pressure analyzed by linear regression. (A) Correlation that includes all groups. (B) Correlation without the $27 \mathrm{G}$ group.

Table 2. Correlations between cardiac hypertrophy and hemodynamic parameters analyzed by stepwise multiple regression.

\begin{tabular}{lcl}
\hline & \multicolumn{3}{c}{$\mathrm{VW} / \mathrm{BW}$} \\
\cline { 2 - 3 } & Pearson correlation $(r)$ & $P$ (1-tailed) \\
\hline Wk-4 SP & 0.951 & 0 \\
Wk-4 DP & 0.712 & 0.002 \\
Wk-4 pulse & 0.343 & 0.115 \\
Wk-4 MAP & 0.835 & 0 \\
Wk-4 HR & -0.123 & 0.337 \\
Wk-4 RPP & 0.833 & 0 \\
Total SP & 0.732 & 0.001 \\
Total DP & 0.545 & 0.022 \\
Total pulse & 0.081 & 0.392 \\
Total MAP & 0.64 & 0.007 \\
Total HR & -0.202 & 0.244 \\
Total RPP & 0.747 & 0.001 \\
\hline VW/BW, ventricular weight/body weight $\left(\mathrm{mg} \mathrm{g}^{-1}\right) ;$ \\
SP, systolic pressure (mmHg); \\
DP, diastolic pressure (mmHg); \\
pulse, pulse pressure (mmHg); \\
MAP, mean arterial pressure (mmHg); \\
HR, heart rate (beats min $\left.{ }^{-1}\right) ;$ \\
RPP, rate-pressure product (mmHg $\times$ beats min $\left.^{-1}\right) ;$ \\
Wk: week. &
\end{tabular}

Cardiac fibrosis correlates with cardiac hypertrophy better than with the systolic pressure. In order to further investigate the relationship between cardiac fibrosis and hypertrophy or POL, we stained the ventricle slices with Mason-trichrome and quantified the fibrosis percentage. As shown by the representative straining (figure 3), greater constriction caused more fibrosis in the hearts. Cardiac fibrosis significantly correlated with the cardiac size (VW/BW), but the correlation with the week-4 average systolic pressure was not significant (table 3). Stepwise multiple regression determined $\mathrm{VW} / \mathrm{BW}$ as the better predictor of cardiac fibrosis. 

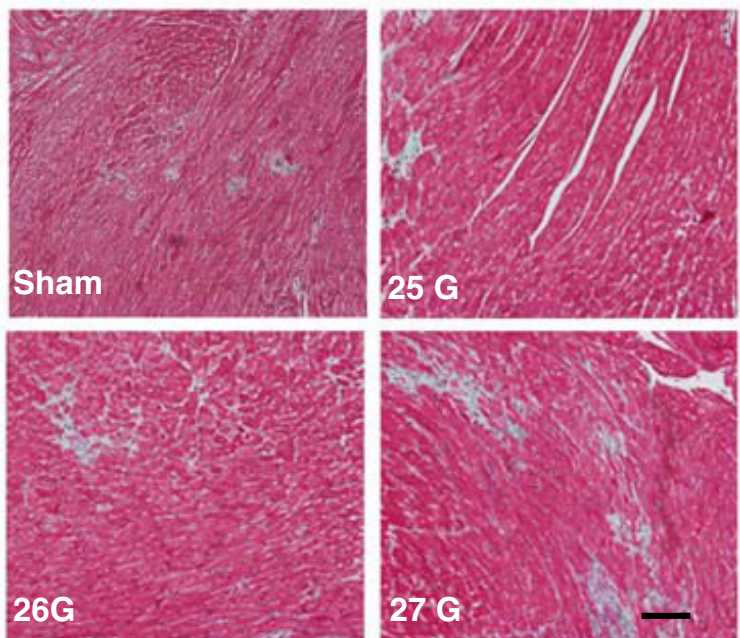

Figure 3. Cardiac fibrosis with aortic constrictions. Representative trichrome-stained transverse sections of left ventricles from sham-operated mice and mice with abdominal aortic constrictions. The bar indicates $100 \mu \mathrm{m}$.

(This figure is in colour only in the electronic version)

Table 3. Correlations between cardiac fibrosis, cardiac hypertrophy and systolic pressure analyzed by stepwise multiple regression.

\begin{tabular}{lll}
\hline & \multicolumn{2}{c}{ Cardiac fibrosis $(\%)$} \\
\cline { 2 - 3 } & Pearson correlation $(r)$ & $P(1$-tailed) \\
\hline VW/BW & 0.59 & 0.013 \\
Wk-4 SP & 0.381 & 0.089 \\
\hline
\end{tabular}

VW/BW, ventricular weight/body weight $\left(\mathrm{mg} \mathrm{g}^{-1}\right)$ $\mathrm{SP}$, systolic pressure $(\mathrm{mmHg})$.

Cardiac gene expression correlates with cardiac hypertrophy and systolic pressure. An altered cardiac gene expression program is one of the characteristics of cardiac hypertrophy, particularly that induced by POL (Schoenfeld et al 1998, Wagner et al 2004). We used RTQPCR to measure some of these expression levels in the ventricles with sham operation or aortic constriction using $25 \mathrm{G}, 26 \mathrm{G}$, or $27 \mathrm{G}$ needles, and correlated them with both VW/BW and week-4 systolic pressure. Expression of ANP (a ventricular embryonic gene), Sk-Actin (a cytoskeletal function related gene) and Coll III (one of the genes that are important for controlling collagen deposition) significantly increased with aortic constriction (data not shown). The expression of PLBN, which is important in calcium handling, decreased with aortic constriction (data not shown). All of these data are consistent with previous reports (Schoenfeld et al 1998, Wagner et al 2004). Normal adult's hearts have a high expression of $\alpha$-MHC and low $\beta$-MHC level, but this is reversed in POL-induced cardiac hypertrophy (Schoenfeld et al 1998, Wagner et al 2004). The expression of $\alpha$-MHC decreased and that of $\beta$-MHC increased with aortic constriction (data not shown), also consistent with previous reports.

The expression of all six genes that we examined significantly correlated with both VW/BW and week-4 systolic pressure (table 4). For the ANP and Sk-Actin expression, the 
Table 4. Correlations between cardiac gene expression and cardiac hypertrophy and systolic pressure analyzed by stepwise multiple regression.

\begin{tabular}{|c|c|c|c|c|}
\hline & \multicolumn{2}{|c|}{$\mathrm{VW} / \mathrm{BW}$} & \multicolumn{2}{|c|}{ wk4 SP } \\
\hline & $\begin{array}{l}\text { Pearson } \\
\text { correlation }(r)\end{array}$ & $\begin{array}{l}P \\
\text { (1-tailed) }\end{array}$ & $\begin{array}{l}\text { Pearson } \\
\text { correlation }(r)\end{array}$ & $\begin{array}{l}P \\
\text { (1-tailed) }\end{array}$ \\
\hline $\mathrm{ANP}^{\mathrm{a}}$ & 0.748 & 0.001 & 0.857 & 0 \\
\hline Sk-Actin ${ }^{\mathrm{a}}$ & 0.731 & 0.001 & 0.784 & 0 \\
\hline$\alpha-\mathrm{MHC}^{\mathrm{a}}$ & -0.684 & 0.003 & -0.554 & 0.02 \\
\hline$\beta-\mathrm{MHC}^{\mathrm{a}}$ & 0.746 & 0.001 & 0.717 & 0.002 \\
\hline $\mathrm{PLBN}^{\mathrm{a}}$ & -0.777 & 0.001 & -0.668 & 0.005 \\
\hline Coll-III $^{\mathrm{a}}$ & 0.767 & 0.001 & 0.615 & 0.01 \\
\hline
\end{tabular}

VW/BW, ventricular weight/body weight $\left(\mathrm{mg} \mathrm{g}^{-1}\right)$;

$\mathrm{SP}$, systolic pressure $(\mathrm{mmHg})$

ANP, atrial natriuretic peptide; Sk-Actin, $\alpha$ skeletal actin;

$\alpha$-MHC, $\alpha$-myosin heavy chain; $\beta$-MHC, $\beta$-myosin heavy chain;

PLBN, phospholamban; Coll-III, type III collagen.

a Gene expression was measured by RT-QPCR and glyceraldehyde-3-phosphate dehydrogenase

(GAPDH) was used as an endogenous control.

correlation with the systolic pressure had higher significance and the week-4 systolic pressure was determined as the predictor by stepwise multiple regression (table 4). However, VW/BW was identified as the predictor for the gene expression of $\alpha$-MHC, $\beta$-MHC, PLBN and Col III (table 4).

\section{Discussion}

Cardiac hypertrophy, fibrosis and gene expression changes are all consequences of POL, a commonly used animal model for cardiovascular research (Rockman et al 1991, De Windt et al 2001, Asakawa et al 2002, McMullen et al 2003, Liao et al 2004, De Acetis et al 2005, Takimoto et al 2005). However, their correlations with direct measurements of hemodynamic parameters in POL have not been determined in conscious ambulatory mice. We demonstrated that these measurements are technically feasible and give tight correlations with cardiac hypertrophy.

Pulsatile arterial pressure in the arch of the aorta was measured via a radiotelemetry device to assess POL in mice after abdominal aortic banding that produced different degrees of constriction. Overall, the cardiac size correlated with the week-4 parameters better than those of all measured periods, which is likely because the mice need 1-2 weeks to regain their circadian rhythm after the telemetry implant surgeries (Leon et al 2004, Whitesall et al 2004). Cardiac hypertrophy correlated best with the week-4 average systolic pressure compared with other hemodynamic parameters such as diastolic pressure, MAP and ratepressure product, suggesting that systolic pressure is the most important determinant of the cardiac size. This finding is consistent with studies in hypertensive human subjects (Lekakis et al 2004, Libhaber et al 2005) that showed cardiac hypertrophy correlated with systolic pressure more significantly.

Previously, Rockman et al measured the degree of constriction (pressure gradient) in anesthetized mice at the end of POL (Esposito et al 2002). The radiotelemetry method allowed the current study to monitor the hemodynamic parameters after POL in conscious mice continuously, which would give more accurate measurements. In addition, the evaluation of the degree of constriction in anesthetized animals cannot account for compensatory changes 
in conscious animals such as changes in vascular resistance or cardiac function that may influence the final POL. For example, a drug that lowers the blood pressure or changes the cardiac function would be expected to alter POL but would have no effect on the degree of constriction. However, the real-time measurement demonstrated in our study will be able to detect these POL changes. In addition to control for degree of constriction, our model will distinguish between primary effects on hearts and effects secondary to changes in the degree of POL when using manipulations that can affect both blood pressure and cardiac myocytes directly. For instance, Asakawa et al used POL model to investigate the effects of thiazolidinediones on cardiac hypertrophy (Asakawa et al 2002). It is well known that thiazolidinediones (Diep et al 2002, Atkins et al 2005) can decrease blood pressure, which makes it unclear if the inhibitory effects observed were because of decreased pressure or intrinsic effects on the hearts. In order to further address the possibly direct effects of these compounds on cardiac hypertrophy, pressure monitoring and correlation to cardiac size would be necessary.

Cardiac fibrosis is a major characteristic of cardiac remodeling in pathological hypertrophy, leading to cardiac dysfunction and failure (Boluyt et al 1994, Heling et al 2000, Kuwahara et al 2002). In this POL-induced cardiac hypertrophy model fibrosis is correlated with the cardiac size but not the blood pressure. One explanation for this is that pressure change is likely not the only factor that affects cardiac fibrosis. Other factors such as inflammation are involved in the cardiac fibrosis process (Nicoletti and Michel 1999, Kuwahara et al 2004, Ogata et al 2004) and inflammation has been observed in hypertensive animal models including the POL model (Nicoletti and Michel 1999, Kuwahara et al 2004, Ogata et al 2004). Further analyzing the correlation between inflammation and fibrosis in this model would lead to more precise explanation on this aspect.

POL-induced cardiac hypertrophy is usually associated with a characteristic pattern of the cardiac gene expression (Schoenfeld et al 1998, Hoshijima and Chien 2002, Wagner et al 2004). This cardiac gene program, including changes in the expression of genes related to growth and metabolism as well as reactivation of fetal genes, may ultimately trigger pathological changes in the myocardium and cause irreversible cardiac dysfunction (Hoshijima and Chien 2002). The significant correlations between the cardiac gene expression and both cardiac size and systolic pressure further demonstrate the accuracy of pressure monitoring to reflect cardiac hypertrophy in our model. For some cardiac genes (ANP and Sk-Actin) their expression correlated with POL better than with the cardiac size, indicating that these genes' expression is dominantly controlled by pressure changes. The expression of other cardiac genes $(\alpha-\mathrm{MHC}$, $\beta$-MHC, PLBN and Coll III) correlated with the cardiac size better than with POL, suggesting that other factors such as inflammation could also contribute to the changes in the cardiac gene expression.

In summary, our data provided more insights about the correlations between blood pressure and other hemodynamic parameters, cardiac hypertrophy, cardiac fibrosis and cardiac gene expression, and demonstrated that direct real-time pressure monitoring is a practical method to extend the usefulness of POL models to manipulations that can affect both blood pressure and myocyte intrinsic responses.

\section{Acknowledgments}

We thank Dr Scott Billecke for critical review of the manuscript and Lingling Zhang for statistical advice. This work was funded in parts by National Heart, Lung, and Blood Institute R01HL070902, R01HL083201 and Program Project Grants 5 P01 HL-1857524. 


\section{References}

Alexander N 1953 Effect of constriction of the abdominal aorta on femoral pulse and mean pressure in rabbits Am. $J$. Physiol. 174 179-84

Asakawa M, Takano H, Nagai T, Uozumi H, Hasegawa H, Kubota N, Saito T, Masuda Y, Kadowaki T and Komuro I 2002 Peroxisome proliferator-activated receptor gamma plays a critical role in inhibition of cardiac hypertrophy in vitro and in vivo Circulation 105 1240-6

Atkins K B, Northcott C A, Watts S W and Brosius F C 2005 Effects of PPAR-gamma ligands on vascular smooth muscle marker expression in hypertensive and normal arteries Am.J. Physiol. Heart Circ. Physiol. 288 H235-43

Boluyt M O, O’Neill L, Meredith A L, Bing O H, Brooks W W, Conrad C H, Crow M T and Lakatta E G 1994 Alterations in cardiac gene expression during the transition from stable hypertrophy to heart failure. Marked upregulation of genes encoding extra cellular matrix components Circ. Res. 75 23-32

Brede M, Wiesmann F, Jahns R, Hadamek K, Arnolt C, Neubauer S, Lohse M J and Hein L 2002 Feedback inhibition of catecholamine release by two different alpha2-adrenoceptor subtypes prevents progression of heart failure Circulation 106 2491-6

De Acetis $\mathrm{M}$ et al 2005 Cardiac over expression of melusin protects from dilated cardiomyopathy due to long-standing pressure overload Circ. Res. 96 1087-94

De Windt L J et al 2001 Targeted inhibition of calcineurin attenuates cardiac hypertrophy in vivo Proc. Natl. Acad. Sci. USA 98 3322-7

Diep Q N, El Mabrouk M, Cohn J S, Endemann D, Amiri F, Virdis A, Neves M F and Schiffrin E L 2002 Structure, endothelial function, cell growth, and inflammation in blood vessels of angiotensin II-infused rats: role of peroxisome proliferator-activated receptor-gamma Circulation 105 2296-302

Duan S Z, Ivashchenko C Y, Russell M W, Milstone D S and Mortensen R M 2005 Cardiomyocyte-specific knockout and agonist of peroxisome proliferator-activated receptor-gamma both induce cardiac hypertrophy in mice Circ. Res. 97 372-9

Esposito G, Rapacciuolo A, Naga Prasad S V, Takaoka H, Thomas S A, Koch W J and Rockman H A 2002 Genetic alterations that inhibit in vivo pressure-overload hypertrophy prevent cardiac dysfunction despite increased wall stress Circulation 105 85-92

Heling A et al 2000 Increased expression of cytoskeletal, linkage, and extra cellular proteins in failing human myocardium Circ. Res. 86 846-53

Hoshijima M and Chien K R 2002 Mixed signals in heart failure: cancer rules J. Clin. Invest. 109 849-55

Kuwahara F, Kai H, Tokuda K, Kai M, Takeshita A, Egashira K and Imaizumi T 2002 Transforming growth factor-beta function blocking prevents myocardial fibrosis and diastolic dysfunction in pressure-overloaded rats Circulation 106 130-5

Kuwahara F, Kai H, Tokuda K, Takeya M, Takeshita A, Egashira K and Imaizumi T 2004 Hypertensive myocardial fibrosis and diastolic dysfunction: another model of inflammation? Hypertension $43739-45$

Lekakis J P et al 2004 Cardiac hypertrophy in hypertension: relation to 24-h blood pressure profile and arterial stiffness Int. J. Cardiol. 97 29-33

Leon L R, Walker L D, DuBose D A and Stephenson L A 2004 Biotelemetry transmitter implantation in rodents: impact on growth and circadian rhythms Am. J. Physiol. Regul. Integr. Comp. Physiol. 286 R967-74

Liao Y et al 2004 Celiprolol, a vasodilatory beta-blocker, inhibits pressure overload-induced cardiac hypertrophy and prevents the transition to heart failure via nitric oxide-dependent mechanisms in mice Circulation $110692-9$

Libhaber E N, Norton G R, Libhaber C D, Candy G P, Woodiwiss A J, Sliwa K, Essop M R and Sareli P 2005 Change in blood pressure predicts regression of cardiac hypertrophy in patients of African ancestry receiving agents influencing the renin-angiotensin system J. Hum. Hypertens. $19659-61$

McMullen J R, Shioi T, Zhang L, Tarnavski O, Sherwood M C, Kang P M and Izumo S 2003 Phosphoinositide 3-kinase(p110alpha) plays a critical role for the induction of physiological, but not pathological, cardiac hypertrophy Proc. Natl. Acad. Sci. USA 100 12355-60

Nicoletti A and Michel J B 1999 Cardiac fibrosis and inflammation: interaction with hemodynamic and hormonal factors Cardiovasc. Res. 41 532-43

Nishida K et al 2004 p38 alpha mitogen-activated protein kinase plays a critical role in cardiomyocyte survival but not in cardiac hypertrophic growth in response to pressure overload Mol. Cell Biol. 24 10611-20

Ogata T, Miyauchi T, Sakai S, Takanashi M, Irukayama-Tomobe Y and Yamaguchi I 2004 Myocardial fibrosis and diastolic dysfunction in deoxycorticosterone acetate-salt hypertensive rats is ameliorated by the peroxisome proliferator-activated receptor-alpha activator fenofibrate, partly by suppressing inflammatory responses associated with the nuclear factor-kappa-B pathway J. Am. Coll. Cardiol. 43 1481-8

Rockman H A, Ross R S, Harris A N, Knowlton K U, Steinhelper M E, Field L J, Ross J Jr and Chien K R 1991 Segregation of atrial-specific and inducible expression of an atrial natriuretic factor transgene in an in vivo murine model of cardiac hypertrophy Proc. Natl. Acad. Sci. USA 88 8277-81 
Schoenfeld J R, Vasser M, Jhurani P, Ng P, Hunter J J, Ross J Jr, Chien K R and Lowe D G 1998 Distinct molecular phenotypes in murine cardiac muscle development, growth, and hypertrophy J. Mol. Cell. Cardiol. 30 2269-80

Takimoto E et al 2005 Oxidant stress from nitric oxide synthase-3 uncoupling stimulates cardiac pathologic remodeling from chronic pressure load J. Clin. Invest. 115 1221-31

Wagner R A, Tabibiazar R, Powers J, Bernstein D and Quertermous T 2004 Genome-wide expression profiling of a cardiac pressure overload model identifies major metabolic and signaling pathway responses $J$. Mol. Cell Cardiol. 37 1159-70

Wettschureck N, Rutten H, Zywietz A, Gehring D, Wilkie T M, Chen J, Chien K R and Offermanns S 2001 Absence of pressure overload induced myocardial hypertrophy after conditional inactivation of Galphaq/Galpha11 in cardiomyocytes Nat. Med. 7 1236-40

Whitesall S E, Hoff J B, Vollmer A P and D'Alecy L G 2004 Comparison of simultaneous measurement of mouse systolic arterial blood pressure by radiotelemetry and tail-cuff methods Am. J. Physiol. Heart Circ. Physiol. 286 H2408-15

Young W G Jr, Sealy W C and Harris J S 1950 Effects of chronic constriction of the thoracic aorta upon renal dynamics Surg. Forum 92 200-5 